\title{
Neural Systems Underlying Decisions about Affective Odors
}

\author{
Edmund T. Rolls, Fabian Grabenhorst, and Benjamin A. Parris
}

\begin{abstract}
Decision-making about affective value may occur after the reward value of a stimulus is represented and may involve different brain areas to those involved in decision-making about the physical properties of stimuli, such as intensity. In an fMRI study, we delivered two odors separated by a delay, with instructions on different trials to decide which odor was more pleasant or more intense or to rate the pleasantness and intensity of the second odor without making a decision. The fMRI signals in the medial prefrontal cortex area 10 (medial PFC) and in regions to which it projects, including the anterior cingulate cortex (ACC) and insula, were higher when decisions were being made compared with
\end{abstract}

\section{INTRODUCTION}

Representations of the affective (reward and punishment) value of many stimuli and events and their subjective correlate pleasantness versus unpleasantness are present in the OFC and the ACC, as shown by neurophysiological, functional neuroimaging, and clinical neuropsychological investigations (Rolls \& Grabenhorst, 2008). These include the reward and punishment value and correlations with the pleasantness/unpleasantness of olfactory (Grabenhorst, Rolls, Margot, da Silva, \& Velazco, 2007; Anderson et al., 2003; Rolls, Kringelbach, \& de Araujo, 2003; Rolls, Critchley, Mason, \& Wakeman, 1996), taste (Grabenhorst, Rolls, \& Bilderbeck, 2008; Small et al., 2003; Rolls, Sienkiewicz, \& Yaxley, 1989), somatosensory (Rolls, O’Doherty, et al., 2003), temperature (Guest et al., 2007), visual (O’Doherty et al., 2003), monetary (Knutson, Rick, Wimmer, Prelec, \& Loewenstein, 2007; O’Doherty, Kringelbach, Rolls, Hornak, \& Andrews, 2001), and social stimuli (Spitzer, Fischbacher, Herrnberger, Gron, \& Fehr, 2007; Moll et al., 2006; Hornak et al., 2003; Kringelbach \& Rolls, 2003). When taking a decision about rewards or punishers, however, a representation of the affective value on a continuous scale may be formed, and that may be followed by a binary (e.g., yes vs. no) decision process about whether to accept or to work for that amount of reward (Rolls, 2008). To make the distinction clear, we may know exactly how pleasant an odor is on a continuous scale, and some part of the brain may represent this, even at the same time that we take the binary decision that it is the sec-

University of Oxford ratings, implicating these regions in decision-making. Decisionmaking about affective value was related to larger signals in the dorsal part of medial area 10 and the agranular insula, whereas decisions about intensity were related to larger activations in the dorsolateral prefrontal cortex (dorsolateral PFC), ventral premotor cortex, and anterior insula. For comparison, the mid orbitofrontal cortex (OFC) had activations related not to decision-making but to subjective pleasantness ratings, providing a continuous representation of affective value. In contrast, areas such as medial area 10 and the ACC are implicated in reaching a decision in which a binary outcome is produced.

ond of two odors that is more pleasant, which could involve separable brain systems. The neuronal representation for the pleasantness or intensity of a stimulus must remain on a continuum of neuronal firing rate, whereas when a decision is taken, the population of neurons reflecting the decision taken goes into a high firing rate state, and the population of neurons representing the other decision is inhibited (de Lafuente \& Romo, 2006; Deco \& Rolls, 2006; Romo, Hernandez, \& Zainos, 2004). Processes such as this transition from spontaneous firing to a binary state of firing of neurons (fast vs. slow) are known to occur in some premotor and related areas such as the macaque ventral premotor cortex when decisions are taken, about in this case which vibrotactile stimulus to choose (Deco, Rolls, \& Romo, 2009; de Lafuente \& Romo, 2006; Deco \& Rolls, 2006; Romo et al., 2004). One of the aims of this investigation is to test whether different brain systems have activity that is differently related to taking a categorical decision versus rating on a continuous scale. It may also be the case that different brain systems are involved in different types of decision, for example, decisions about affective value versus decisions about physical properties of stimuli such as their intensity, and it was an aim to investigate this also.

To investigate these issues, we performed an fMRI study in which the same set of stimuli was used in different trials, but on some trials only the affective value and intensity had to be rated on a continuous scale, whereas on other trials binary decisions had to be made about whether the second stimulus was more or less pleasant than the first or on other trials about whether the second stimulus was more of less intense than the first. We used olfactory 
stimuli because many of them have affective value (i.e., are pleasant or unpleasant) and because they can be delivered with a short delay between them in a simple decision task of whether the first or second stimulus is more pleasant or, on other trials, is more intense. On the rating trials, the identical set of stimuli was presented, but only ratings, and no decisions, were required. The different trial types were identical up to 1 sec before the time of presentation of the second odor, at which time a visual instruction appeared to, on that trial, rate, decide on pleasantness, or decide on intensity.

Previous investigations of decision-making have not directly addressed the issue of how activity in different brain systems may be related to representing affective value, intensity, or taking decisions about intensity versus affective value. However, previous studies have identified contributions of the parietal cortex (Sugrue, Corrado, \& Newsome, 2005; Glimcher, 2003), dorsolateral prefrontal cortex (DLPFC; Hampton \& O’Doherty, 2007; Knutson et al., 2007; Heekeren, Marrett, Bandettini, \& Ungerleider, 2004), OFC (Hornak et al., 2004; Kringelbach \& Rolls, 2003; Bechara, Damasio, \& Damasio, 2000; Zatorre, JonesGotman, \& Rouby, 2000), cingulate cortex (Behrens, Woolrich, Walton, \& Rushworth, 2007; Marsh, Blair, Vythilingam, Busis, \& Blair, 2007), and ventral premotor cortex (Deco \& Rolls, 2006; Romo et al., 2004) to decision-making.

\section{METHODS}

\section{Design}

The aim of the design was described in the Introduction. The design is based on the vibrotactile decision-making studies of Romo et al. (2004) in which vibrotactile stimuli separated by a delay were presented and a decision had to be made when the second stimulus was presented of whether the second frequency was higher than the first. Our design was extended by having two different types of decision on contrasting trials, about whether the second odor was more pleasant or more intense than the first, to enable systems involved in different types of decision to be compared, and by having a condition on which identical stimuli were presented to those on the decision trials, but no choice decision was required, only a rating on a continuous scale of the pleasantness and intensity to allow brain systems involved in taking decisions to be contrasted with those involved in rating the values on a continuous scale of the identical stimuli.

Because the human participants made ratings of the pleasantness and intensity of the stimuli on some of the trials during the fMRI investigation, we were able to analyze how their subjective feelings of pleasantness and intensity were related to activations in different brain regions. The design allowed us to investigate not only where the affective value and intensity of the olfactory stimuli are represented but also how the brain systems that represent the affective and intensity value respond when binary decisions are made about the stimuli. The design also allowed investigation of whether the brain areas that have activity related to the different types of decision-making (about pleasantness or about intensity) also have activity that reflects the affective value or the intensity of the stimuli as shown by correlations with the subjective ratings that were made to the stimuli.

On decision trials, the task required a binary decision starting during the second odor about which of the two odors was more pleasant or (on different trials, as indicated by the instruction at $t=7 \mathrm{sec}$ ) more intense. The first odor was presented for $2 \mathrm{sec}$ at $t=0 \mathrm{sec}$ on a trial, the second odor for $2 \mathrm{sec}$ at $t=8 \mathrm{sec}$, and from the start of the second odor there was a decision period until $t=$ $16 \mathrm{sec}$ when the participants could respond by pressing a key of a button box (see Figure 1). The 1-sec instruction of the trial type appeared at $t=7 \mathrm{sec}$ and indicated "decide pleasantness" or "decide intensity." Subjects were instructed to make the decision required as soon after the second odor as possible, that is, early in the decision period.

The rating trials including the odors were identical until the time of the instruction at $t=7 \mathrm{sec}$, which instead read "rate stimulus" (ensuring no difference between the rate and decision trials until $t=7 \mathrm{sec}$, as shown in Figure 1). At $t=16 \mathrm{sec}$ of these trials, rating scales for pleasantness and intensity were shown, and the same button box response keys were used to make the ratings as to make the responses on the decision trials. The rating was of the pleasantness and intensity of the second odor. As shown in Figure 1, the structure of the rating and the binary decision trials was very similar, and in both cases the response could not be made until $t=16 \mathrm{sec}$. The fMRI analysis of the difference in the neural processing on these different trial types started at $t=8 \mathrm{sec}$ and used the standard hemodynamic response function, so that differences in the processing related to deciding versus rating at $t=$ $8 \mathrm{sec}$ were what was measured in the statistical analysis. On both rating and decision trials, the first stimulus had to be remembered until $t=7 \mathrm{sec}$ when the instruction came that this was a decision or a rating trial, and the second odor was delivered at $t=8 \mathrm{sec}$, and that was the time when on decision trials a choice decision could be reached about whether the second odor was more or less pleasant and more or less intense than the first odor, whereas on rating trials the continuous affective value of the second odor had to be processed. Thus, the major feature that distinguished the decision and rating trials was making a choice versus processing a value on a continuous scale, with other factors such as the prior short-term memory processing, etc., held as similar as possible.

The plan of the design was to enable processes involved in the affective representation of sensory (olfactory) stimuli to be compared with decision-making about the same affective stimuli. Brain mechanisms involved in decisionmaking might involve decoding an affective representation of a stimulus and then taking a decision about the stimulus, and the design was to allow investigation of 
Figure 1. Task design for trials of the olfactory task. On decision trials (top), the task required a binary decision starting during the second odor about which of the two odors was more pleasant or (on different trials, as indicated by the instruction at $t=7 \mathrm{sec}$ ) more intense. On rating trials (bottom), identical stimuli were used, but no decision was required, and instead participants rated the second odor for pleasantness and intensity on continuous analog visual rating scales. The trial types were identical until $t=7 \mathrm{sec}$, when the instruction indicated whether the trial type was decide or rate. The second odor was delivered at $t=8 \mathrm{sec}$, the subjects were deciding or rating at that time, and the imaging was with respect to this period starting at $t=8 \mathrm{sec}$. No responses to indicate the decision made or the rating value could be made until $t=16 \mathrm{sec}$.

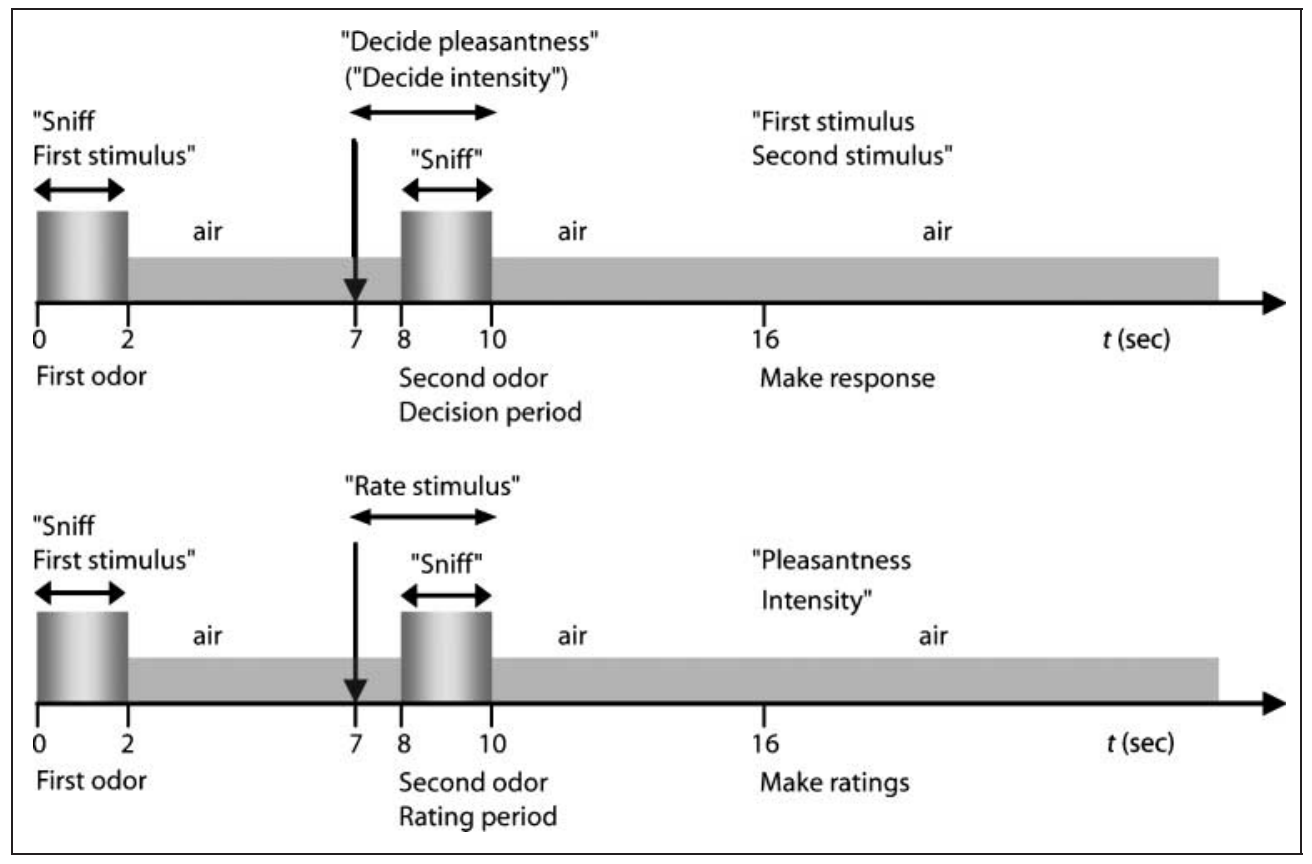

what extra processes might be involved in taking the decision as compared with only representing the affective value.

The olfactory stimuli were delivered through an olfactometer, and the pleasant stimuli were citral and vanillin, whereas the unpleasant stimuli were hexanoic acid and isovaleric acid.

We investigated how the decision-making and the affective value of the olfactory stimuli were represented in brain areas identified by prior hypotheses such as the OFC, where the pleasantness and unpleasantness of olfactory stimuli are represented (Grabenhorst et al., 2007; de Araujo, Rolls, Velazco, Margot, \& Cayeux, 2005; Anderson et al., 2003; Rolls, Kringelbach, et al., 2003) in medial PFC area 10, insula, DLPFC, ACC, ventral premotor cortex, and ventral tegmental area where activations are found in some decision-making tasks (Grabenhorst, Rolls, \& Parris, 2008; Burgess, Dumontheil, \& Gilbert, 2007; Rushworth, Behrens, Rudebeck, \& Walton, 2007; Thielscher \& Pessoa, 2007; Morris, Nevet, Arkadir, Vaadia, \& Bergman, 2006; Heekeren et al., 2005; Romo et al., 2004; Kim \& Shadlen, 1999). We report results for all of these a priori regions in this article and show in the Supplementary material the activations in these and other areas in global contrast and correlation analyses.

\section{Participants}

Twelve healthy volunteers ( 7 men and 5 women, mean age $=27$ years) participated in the study. The study was conducted in accord with the Declaration of Helsinki and approved by the Central Oxford Research Ethics Committee, and written informed consent from all subjects was obtained before the experiment.

\section{Stimuli}

The set of olfactory stimuli used was selected based on previous fMRI studies on olfaction (Rolls, Kringelbach, et al., 2003). The pleasant odors were $1 \mathrm{M}$ citral and $4 \mathrm{M}$ vanillin. The unpleasant odors were hexanoic acid (10\% v/v) and isovaleric acid (15\%). The odors were made up in propylene glycol.

\section{Stimulus Delivery}

A purpose-built continuous airflow 10-channel computercontrolled olfactometer was used to allow odor stimuli to be delivered in the MRI scanner (Rolls, Kringelbach, et al., 2003). The control and the metal components of the system are kept outside the scanner room, and the system is free of any auditory, tactile, or thermal shifts that could cue the subject to the onset of odor delivery. The flow of clean medical air is controlled using a pressure regulator and flow meter. The air is directed using solenoidoperated valves controlled by the stimulus computer using transistor-transitor logic (TTL) pulses to either a clean air washbottle containing only solvent or propylene glycol or to one of four other washbottles each containing one odorant dissolved in the propylene glycol. Each washbottle is 
connected by its own Teflon tube (to provide for low adhesion) to a single delivery nozzle placed within $1 \mathrm{~cm}$ of the nose to minimize dead space. The delivery nozzle provided two tubes, one for each nostril, to produce birhinal stimulation. The flow rate of the air supply was kept constant at 2 $\mathrm{L} / \mathrm{min}$ such that the same minimal degree of tactile somatosensory stimulation was delivered throughout. The air line was on continuously by default and was switched off only when the solenoid directed the clean air supply to another washbottle so that an odorant could be delivered. This resulted in a system with no perceptible pressure change when the air was replaced during stimulus delivery by an odor for $2 \mathrm{sec}$. This system was used in previous fMRI studies of human olfaction (Rolls, Kringelbach, et al., 2003)

\section{Experimental Protocol}

The experimental protocol consisted of an event-related interleaved design presenting in random permuted sequence the three experimental conditions and different pairs of olfactory stimuli for each condition. Each trial started at $t=0 \mathrm{sec}$ with the first odor being delivered for $2 \mathrm{sec}$ accompanied by a visual label stating "sniff first stimulus" (see Figure 1). There was then a 6-sec period during which clean air was delivered. In this period at $t=$ 7 sec, a visual label was displayed stating either "decide pleasantness," "decide intensity," or "rate stimulus." At $t=$ $8 \mathrm{sec}$, the second odor was presented for 2 sec accompanied by a visual label stating either "sniff decide pleasantness," "sniff decide intensity," or "sniff rate stimulus." There was then a 6-sec period during which clean air was delivered. Starting at $t=16 \mathrm{sec}$ on decision trials, the words "first stimulus" and "second stimulus" appeared on the screen for $2 \mathrm{sec}$, and in this period, the participant had to select which button key response to make (up button or down button) for the decision that had been taken at the time when the second odor was delivered. At $t=18 \mathrm{sec}$ on decision trials, the words "first stimulus" or "second stimulus" were then displayed to provide feedback to the participant that their choice was acknowledged. On rating trials, starting at $t=16 \mathrm{sec}$, the subjective ratings were made. The first rating was for the pleasantness of the second odor on a continuous visual scale from -2 (very unpleasant) through 0 (neutral) to +2 (very pleasant). The second rating was for the intensity of the second odor on a scale from 0 (very weak) to 4 (very intense). The ratings were made with a visual rating scale in which the subject moved the bar to the appropriate point on the scale using a button box. There was $4 \mathrm{sec}$ for each rating. Subjects were pretrained outside the scanner in the whole procedure and use of the rating scales. Each of the trial types was presented in random permuted sequence 36 times. Which two of the four odors were presented on each trial and the order in which they were presented was determined by a random permuted sequence. This general protocol and design has been used successfully in previous studies to investigate activations and their relation to subjective ratings in cortical areas (Grabenhorst, Rolls, \& Bilderbeck, 2008; Grabenhorst et al., 2007; de Araujo et al., 2005; Rolls, Kringelbach, et al., 2003; Rolls, O’Doherty, et al., 2003).

\section{fMRI Data Acquisition}

Images were acquired with a 3.0-T VARIAN/SIEMENS whole-body scanner at the Centre for Functional Magnetic Resonance Imaging at Oxford (FMRIB), where 27 T2*weighted EPI coronal slices with an in-plane resolution of $3 \times 3 \mathrm{~mm}$ and between plane spacing of $4 \mathrm{~mm}$ were acquired every $2 \mathrm{sec}$ (repetition time $=2 \mathrm{sec}$ ). We used the techniques that we have developed over a number of years (de Araujo, Kringelbach, Rolls, \& Hobden, 2003; O’Doherty, Rolls, Francis, Bowtell, \& McGlone, 2001), and as described in detail by Wilson et al. (2002), we carefully selected the imaging parameters to minimize susceptibility and distortion artifact in the OFC. The relevant factors include imaging in the coronal plane, minimizing voxel size in the plane of the imaging, as high a gradient switching frequency as possible $(960 \mathrm{~Hz})$, a short echo time of $28 \mathrm{msec}$, and local shimming for the inferior frontal area. The matrix size was $64 \times 64 \mathrm{~mm}$ and the field of view was $192 \times 192 \mathrm{~mm}$. Continuous coverage was obtained from +62 (A/P) to $-46(\mathrm{~A} / \mathrm{P})$. A whole-brain T2*-weighted EPI volume of the above dimensions and an anatomical T1 volume with a coronal plane slice thickness of $3 \mathrm{~mm}$ and in-plane resolution of $1 \times 1 \mathrm{~mm}$ were also acquired.

\section{fMRI Data Analysis}

The imaging data were analyzed using SPM5 (Statistical Parametric Mapping; Wellcome Institute of Cognitive Neurology, London). Preprocessing of the data used SPM5 realignment, reslicing with sinc interpolation, normalization to the MNI coordinate system (Montreal Neurological Institute; Collins, Neelin, Peters, \& Evans, 1994), and spatial smoothing with a 6-mm FWHM isotropic Gaussian kernel. The time series at each voxel were low-pass filtered with a hemodynamic response kernel. Time series nonsphericity at each voxel was estimated and corrected for (Friston et al., 2002), and a high-pass filter with a cutoff period of 128 sec was applied. In the single event design, a general linear model (GLM) was then applied to the time course of activation where the decision or rating period onsets $(t=8$ sec in each trial) were modeled as single impulse response functions and then convolved with the canonical hemodynamic response function (Friston, Worsley, Frackowiak, Mazziotta, \& Evans, 1994). Linear contrasts were defined to test specific effects. Time derivatives were included in the basis functions set. Following smoothness estimation (Kiebel, Poline, Friston, Holmes, \& Worsley, 1999), in the 
first stage of analysis, condition-specific experimental effects (parameter estimates or regression coefficients pertaining to the height of the canonical HRF) were obtained via the GLM in a voxelwise manner for each subject. The results were obtained in the following GLM models: (a) regressors of all decide trials and rate trials at the time when the second odor was presented and of button box responses made in the response period (used for the main analysis of decide vs. rate); (b) regressors of pleasantnessdecide, intensity-decide, and rating trials at the time when the second odor was presented; and (c) separate regressors for the pleasantness and the intensity ratings on rating trials. Voxel values for each contrast resulted in a statistical parametric map of the corresponding $t$ statistic, which was then transformed into the unit normal distribution $(\mathrm{SPM} z)$. The statistical parametric maps from each individual's data set were then entered into second-level (group), random effects analyses accounting for both scan-to-scan and subject-to-subject variability. More precisely, the sets of individual statistical maps corresponding to a specific effect of interest were entered as covariates in multiple regression models as implemented in SPM5, and the corresponding group effects were assessed by applying linear contrasts (again following smoothness estimation) to the (second-level) parameter estimates generating a $t$ statistics map for each group effect of interest. The correlation analyses of the fMRI BOLD signal with given parameters of interest (e.g., the pleasantness ratings) were performed at the second level through applying one-sample $t$ tests to the first-level statistical parametric maps resulting from performing linear parametric modulation as implemented in SPM5. (We use the term BOLD signal to refer to the level of signal from the BOLD effects that are measured by fMRI.) We report results for brain regions where there were prior hypotheses as described in the Design section and applied small volume (false discovery rate) corrections for multiple comparisons (Worsley, Marrett, Neelin, Friston, \& Evans, 1996) with a radius corresponding to the FWHM of the spatial smoothing filter used. Peaks are reported for which $p<.05$, although the exact corrected probability values (Worsley et al., 1996) are given in the text. In addition to the conventional statistical criterion just described for a significant effect calculated for the peak voxel of a region of activation, we show in Table 1 of the Supplementary material the results of global contrast and correlation analyses using criteria of $p<.0001$ or $p<.001$ uncorrected for multiple comparisons and a cluster threshold of $k=3$, and these additional statistics confirmed the same effects in the a priori regions.

The global SPM analyses consisted of the following: the contrasts of all trials on which decisions were made-all trials on which ratings were made (all-decide - rate); of all trials on which pleasantness decisions were made-all trials on which ratings were made (pleas-decide - rate); of all trials on which intensity decisions were made-all trials on which ratings were made (intens-decide - rate); of all trials on which pleasantness decisions were made-all tri- als on which intensity decisions were made (pleas-decide intens-decide); of all trials on which intensity decisions were made-all trials on which pleasantness decisions were made (intens-decide - pleas-decide); and correlation analyses between the BOLD signal and the pleasantness ratings and the intensity ratings. When contrasts were made between pleasantness decision and intensity decision trials, the contrast (all-decide - rate) was significant in most cases (except where discussed below), so that the area was implicated by this in choice decision-making.

For the time course plots, we located activations within the a priori ROIs and extracted event-related responses from the peak voxel for that subject using the fitted responses provided by SPM, which reflect the hemodynamic response function and are baseline adjusted. These single-subject time courses were then averaged across subjects.

\section{RESULTS \\ Behavioral Results for the Ratings and the Decisions}

The affective (pleasantness) ratings of the odor stimuli across subjects were (on a scale of $+2=$ very pleasant to $-2=$ very unpleasant; mean \pm SEM) $1.1 \pm 0.1$ for citral, $1.2 \pm 0.1$ for vanillin, $-0.9 \pm 0.2$ for hexanoic acid, and $-0.5 \pm 0.2$ for isovaleric acid, $F(3,44)=46.3, p<<$ .001 . Consistent with these ratings, when making pleasantness decisions, the subjects chose, on average, the citral $68.4 \pm 11.4 \%$ of the time over the other odors, the vanillin $85.2 \pm 6.1 \%$ of the time, the hexanoic acid $17.2 \pm 7.9 \%$ of the time, and the isovaleric acid $29.2 \pm$ $10.9 \%$ of the time. The intensity ratings of the odor stimuli across subjects were (on a scale of $+4=$ very intense to $0=$ very weak) $2.4 \pm 0.2$ for citral, $1.4 \pm 0.2$ for vanillin, $2.1 \pm 0.2$ for hexanoic acid, and $1.6 \pm 0.2$ for isovaleric acid, $F(3,44)=5.03, p<.01$. Consistent with these ratings, when making intensity decisions, the subjects chose, on average (as above), the citral 71.5 $\pm 7.4 \%$ of the time over the other odors, the vanillin $26.7 \pm 7.4 \%$ of the time, the hexanoic acid $56.7 \pm 11.3 \%$ of the time, and the isovaleric acid $36.3 \pm 7.8 \%$ of the time.

\section{Medial Prefrontal Cortex Area 10}

A contrast of all trials on which decisions were made versus all trials on which ratings were made (abbreviated as all-decide - rate) revealed an extensive region of differential BOLD signal in medial PFC area 10 with peak at [2 $50-12$ ] $, z=3.78, p<.001$ (pink circle, at cursor), as shown in Figure 2A. The region was anterior to the pregenual cingulate cortex. These signals were measured at identical times in the two trial types, at $t=8 \mathrm{sec}$, which is the time at which the second odor stimulus was delivered and the participant had on decision trials to take a 
decision about whether it was the first or the second stimulus that was more pleasant (on pleasant-decide trials) or more intense (on intensity-decide trials) or on rating trials had to remember and rate the second stimulus (see Figure 1). The time courses of the signals in this medial PFC area 10 region are shown in Figure $2 \mathrm{~B}$ and confirm that the signal was greater on both types of decision trials than on rating trials. All time courses shown in the figures are aligned with respect to the onset of the second odor. The SPM regression analysis showed that there were no significant correlations with the pleasantness or intensity ratings in this region, as addressed further below. In Fig- ure $2 \mathrm{~A}$, an exclusive mask with activations related to motor responses illustrated in Figure 7 was applied. A somewhat more dorsal part of medial area 10 also showed effects in the contrast of all-decide - rate ([8 5010$], z=3.93, p=$ .001 ; see Figure 2A, above the pink circle).

An SPM analysis performed on the decision trials in which the regressor was the absolute value of each subject's average difference in the intensity of each pair of stimuli (as obtained from the rating trials) showed a significant positive correlation at medial PFC site indicated by the pink circle in Figure 2A ( $\left[\begin{array}{lll}-4 & 58 & 0\end{array}\right], z=2.97$, $p<.04)$. Consistently, the percent BOLD signal in this
Figure 2. (A) A contrast of all trials on which decisions were made versus all trials on which ratings were made. An extensive region with more BOLD signal in this contrast was found in medial area 10 with peak at [2 $50-12], z=3.78$, $p<.001$ (pink circle, at cursor). (B) The time course of the BOLD signal in medial area 10 (mean across subjects $\pm S E M$ ) for the different conditions, decide about whether the second odor is more pleasant (pleas-decide), decide about whether the second odor is more intense (intens-decide), and rate the pleasantness and the intensity without any choice (rate). Significant effects are also found in dorsal ACC, [6 28 12] $, z=4.09, p<.001$ (white circle), with time courses in panel $\mathrm{C}$, and in the medial OFC, [8 $36-28$ ], $z=4.40$, $p<.001$ (yellow circle), with time courses in panel D. An exclusive mask with activations related to motor responses illustrated in Figure 7 was applied. The time courses start at the time at which the second odor was presented. (E) The percent BOLD signal in medial area 10 on the decision trials was correlated with the absolute value of the difference between the intensity ratings made to the same set of stimuli on the rating trials $(r=0.81, d f=6$, $p<.03)$. The relation shows that the activations were correlated with the easiness of the decision. (The percent BOLD values were calculated by obtaining the average \pm SEM BOLD signal for intensity ratings binned at increments of 0.25 for each subject and then averaging across subjects.)
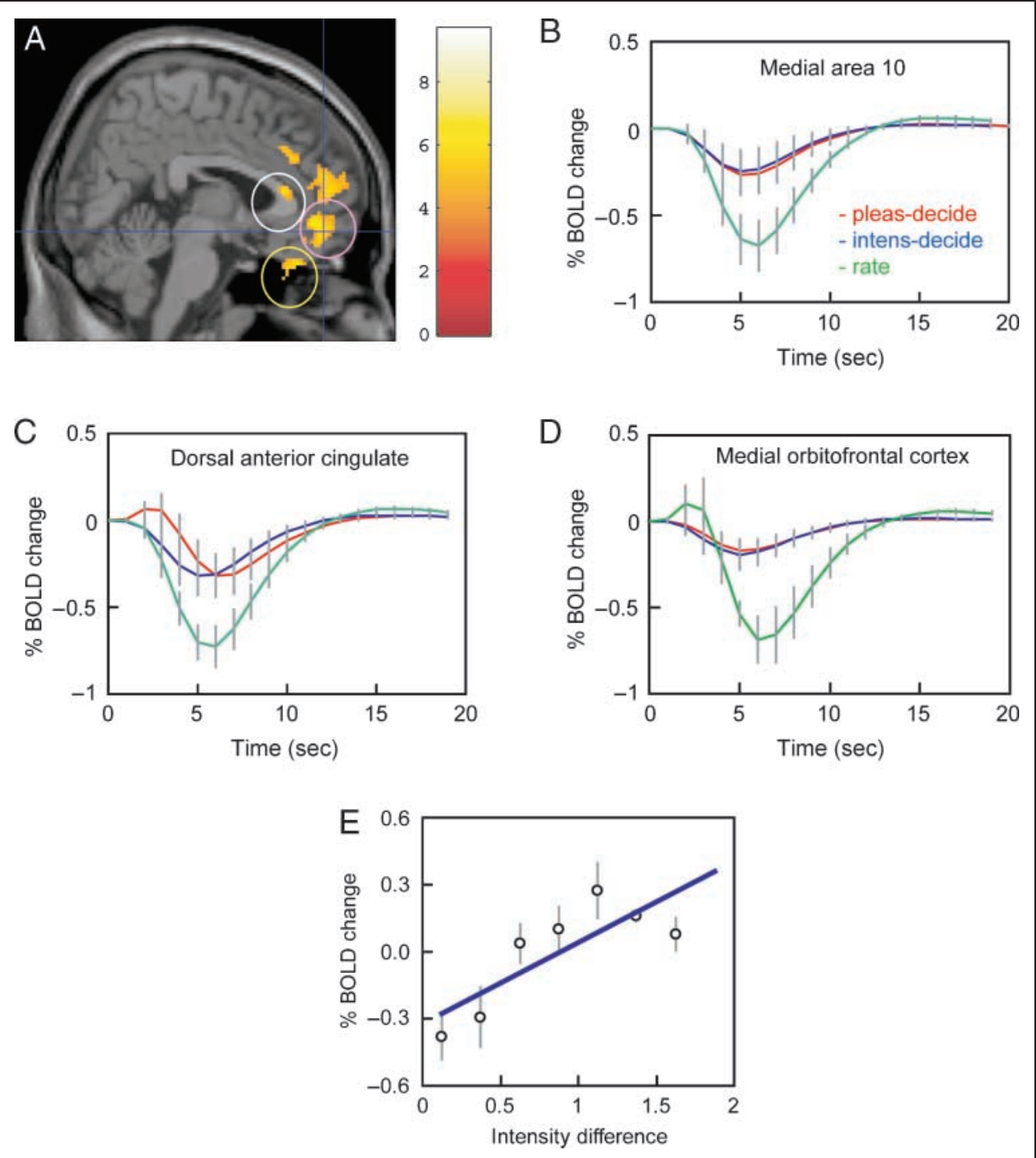
Figure 3. (A) A region of the mid OFC where there was a significant correlation in the SPM regression analysis with the pleasantness ratings ([ $\left.\begin{array}{llll}40 & 36 & -12]\end{array}\right], z=3.13$, $p=.024)$. (B) The relation between the BOLD signal and the pleasantness ratings $(r=0.75, d f=15, p<.001)$. This graph was produced by taking for each subject the average of the BOLD response in the peak voxel (as in previous analyses; Grabenhorst, Rolls, \& Bilderbeck, 2008; Rolls et al., 2008; Grabenhorst et al., 2007; Lau \& Passingham, 2007) in the three time bins at 4, 6, and $8 \mathrm{sec}$ poststimulus, on each trial, and the corresponding rating. For each subject, the means were calculated in discretized ranges of the rating function (e.g., -2 to -1.75 , -1.75 to -1.5 , etc.). These values were then averaged across subjects. (C) The time course of the BOLD signal changes, which start as in the other figures at the time at which the second odor was presented. (D) The SPM parameter estimates for this OFC region and for medial prefrontal cortex area 10 in Figure 2 for the pleasantness correlation and for the decide versus rate contrast. An ANOVA on these parameter estimates revealed a very significant interaction, $F(1,11)=16.0, p=.002$.

region on the decision trials was correlated with the absolute value of the difference between the intensity ratings made to the same set of stimuli on the rating trials $(r=0.81, d f=6, p<.03$; Figure $2 \mathrm{E})$. The relation shows that the activations were larger when binary decisions about large differences in intensity were being made than when the intensities were more similar; that is, the activations were correlated with the easiness of the decision.

A more dorsal part of medial PFC area 10 had a higher BOLD signal when the decisions were about pleasantness than when they were about intensity (as shown by a significant effect in the contrast pleas-decide - intensdecide, [0 62 18], $z=3.12, p=.02$, see Figure 4B, and as illustrated in the dorsal medial prefrontal cortex time courses shown in Figure 4D). (This area is implicated by the present findings in decision-making about pleasant odors rather than just general processing of pleasant odors because its activations were not correlated with the pleasantness ratings of the stimuli, as shown in Table 1 of the Supplementary material.) This is of interest because some other brain areas considered below (including DLPFC; see Figure 4A) showed more signal when the decisions were about intensity than pleasantness. We note that the dorsal part of medial area 10 has been implicated in self-referential processing (Ochsner et al., 2004), and this may be relevant when a subjective preference choice between two affective odors has to be made.

\section{OFC: Areas Where There Was a Correlation with the Pleasantness Ratings But No Effect of Decision-making}

Figure $3 \mathrm{~A}$ shows a region of the mid OFC where there was a significant correlation in the SPM regression analysis with the pleasantness ratings ([40 $36-12], z=3.13$, $p=.024)$. Figure $3 \mathrm{~B}$ shows that the BOLD signal was clearly related to the pleasantness ratings $(r=0.75$, $d f=15, p<.001)$. This brain region had no significant effect in the SPM contrast decide - rate, and this is further illustrated by the similar time courses for the rating and decision conditions in Figure 3C. Thus, activations in this part of the OFC reflected the continuous pleasantness value of the olfactory stimuli and not the binary decision about whether the current stimulus odor was more pleasant or less pleasant than the first.

To test whether there was a dissociation between this part of OFC and medial PFC area 10 as shown in Figure 2 at $[250-12]$, we show in Figure 3D the SPM parameter estimates for these two brain regions for the pleasantness correlation and for the decide versus rate contrast. An ANOVA on these parameter estimates revealed a very significant interaction, $F(1,11)=16.0, p=$ .002 . Thus, these results show that medial PFC area 10 is relatively more related to the decision-making, and this part of the OFC is relatively more related to pleasantness ratings. Indeed, the parameter estimates in Figure 3D show that there is little effect of decision-making in this 
Figure 4. (A) A contrast of decisions about intensitydecisions about pleasantness (intens-decide - pleas-decide) revealed effects in DLPFC ([30 50 36], $z=3.24, p<.05$ ), with the time course shown in panel C. (B) A contrast of decisions about pleasantnessdecisions about intensity (pleas-decide - intens-decide) revealed effects in the dorsal part of medial area 10 (above the area circled in pink in Figure 2A) at [0 62 18], $z=$ $3.12, p=.02$, with the time course shown in panel $\mathrm{D}$. (E) There was a significant interaction between the SPM parameter estimates (arbitrary units) for the signals in medial area 10 versus DLPFC when taking decisions about pleasantness versus intensity, $F(1,11)=66.8, p=5 \times 10^{-6}$. Conventions as in Figure 2.
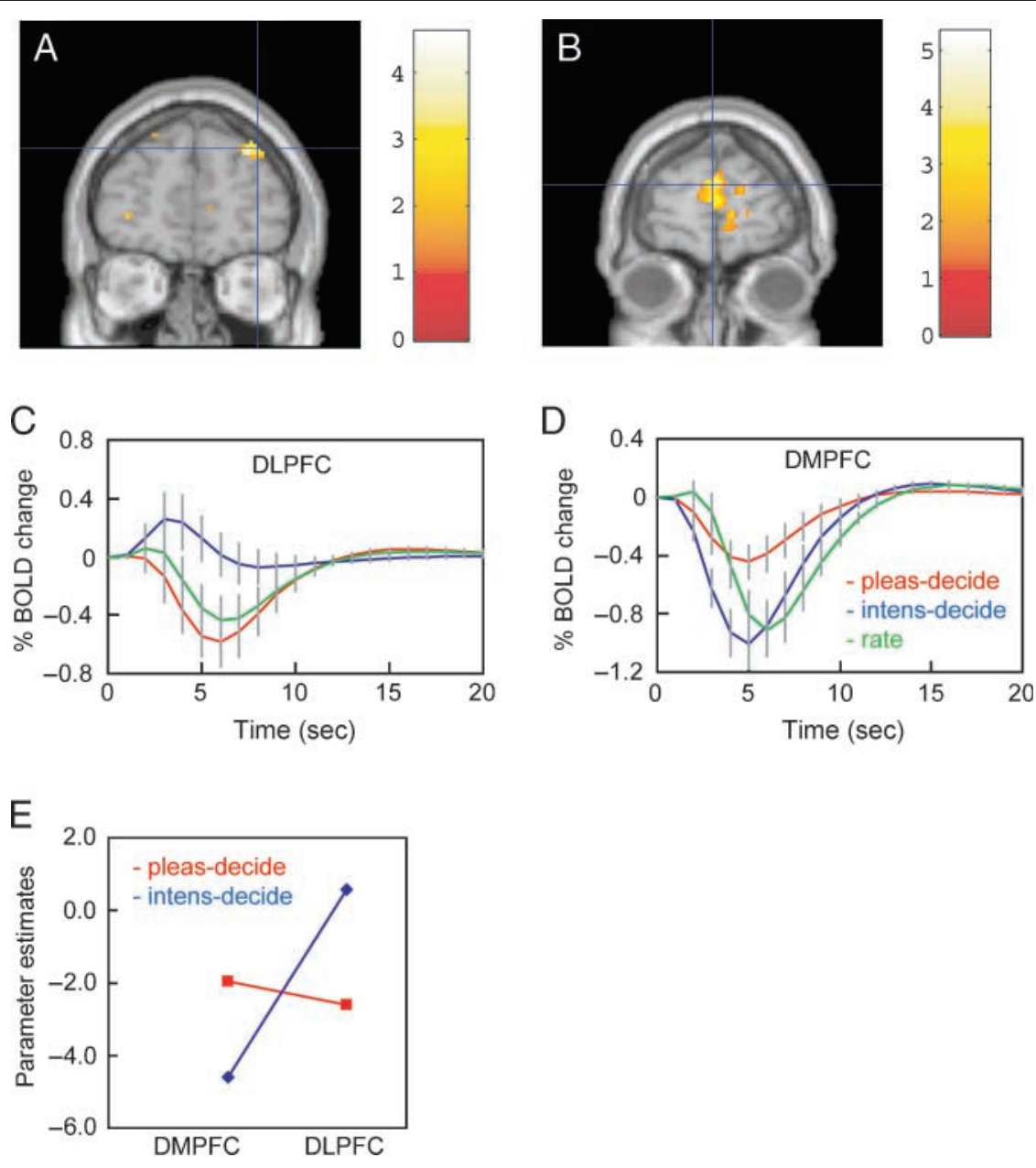

part of OFC (with parameter estimates close to 0 ) and that there is little effect of the pleasantness value in medial PFC area 10 (with parameter estimates close to 0). A part of (anterior) medial OFC also had activations that

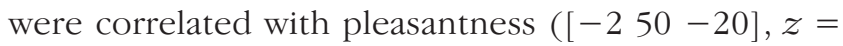
$3.27, p=.035$ ) but had no significant effects in relation to decision-making.

\section{OFC: Areas Where There Was an Effect of Decision-making But No Correlation with the Pleasantness Ratings}

Significant effects of the contrast decide - rate were found in an area that extended ventrally from the area 10 region described above into the medial OFC at [8 $36-28$ ], $z=$ $4.40, p<.001$ (Figure 2A, yellow circle, with time courses shown in Figure 2D). This part of the OFC did not have activations that were significantly correlated with the pleasantness ratings. Two further similar effects in this contrast were found in the posterior OFC ([8 14 -24] and [ $\begin{array}{lll}-32 & 18\end{array}$ $-22]$; for further details, see Supplementary material). Thus, some parts of the OFC are affected by the binary decision-making about odors.

\section{Dorsolateral Prefrontal Cortex}

A contrast of all trials on which decisions were made versus all trials on which ratings were made revealed significant effects in the DLPFC (e.g., at [26 58 30], $z=3.78, p=$ $.001)$, very close to the region shown in Figure 4A. This region appeared to be specially related to decisions about intensity relative to decisions about pleasantness in that as shown in Figure 4A there was a significant effect with the contrast intens-decide - pleas-decide ([30 50 36], $z=3.24$, $p<.05)$. The activation is illustrated by the DLPFC time course in Figure 4C. Moreover, the parameter estimates shown in Figure $4 \mathrm{E}$ for the effects related to these two trial types were different for DLPFC and dorsal medial prefrontal area 10 region illustrated in Figure 4B (ANOVA interaction), $F(1,11)=66.8, p=5 \times 10^{-6}$. This is an indication that the signal in DLPFC when compared with that in medial area 10 is more related to decisions about intensity compared with decisions about pleasantness. (This area is implicated by the present findings in decisionmaking about the intensity of odors rather than just general processing of the intensity of odors because its activations were not correlated with the intensity ratings of the stimuli, as shown in Table 1 of the Supplementary material. 
Moreover, as shown in Figure 4, this area was significantly more activated on intensity decision trials than on either pleasantness decision trials or rating trials.)

The DLPFC region showed more activation on difficult than on easy trials, consistent with the possibility that difficult decisions require more attention. In particular, an SPM analysis performed on the decision trials in which the regressor was the absolute value of each subject's average difference in the intensity of each pair of stimuli (as obtained from the rating trials) showed a significant negative correlation in this DLPFC region ([42 20 50], $z=$ $3.01, p=.023)$. A corresponding effect was found for pleasantness decisions when the regressor was the absolute value of each subject's average difference in pleasantness ([30 52 32], $z=3.05, p=.030)$.

\section{Insula}

Activations were found in the insula that related its activity to different types of decision. A far anterior part of insula, agranular insula, had activation in the contrast pleas-decide - rate ([48 26 4], $z=3.69, p=.002$; Figure $5 \mathrm{~A}$, with time courses illustrating this in Figure $5 \mathrm{C}$ ), implicating agranular insula particularly in decisions about the affective properties of stimuli, such as their pleasantness (similar effects were not found for intensity). An anterior part of insula was activated in the contrast intens-decide - rate (Figure 5B), implicating this part of insula particularly in decisions about the physical properties of stimuli, such as intensity (similar effects were not found for pleasantness). Indeed, a dissociation of these parts of the insula was found, with agranular insula showing relatively more activation for decisions about pleasantness and the anterior insula relatively more activation for decisions about intensity (ANOVA interaction), $F(1,11)=15.3, p=.002$ (see Figure 5E).

The activation of the insular region was related to task difficulty as shown by the following. An SPM analysis performed on the decision trials in which the regressor was the absolute value of each subject's average difference in the pleasantness of each pair of stimuli (as obtained from the rating trials) showed a significant negative correlation in this agranular insular region ([ $\left.\begin{array}{llll}4 & 6 & 20 & 2\end{array}\right], z=2.87$, $p=.011)$. These greater activations for difficult decisions could be related to affective concomitants of such decisions because this region is known to have activations
Figure 5. Decisions about intensity compared with decisions about pleasantness. (A) The agranular insula showed significant effects in the contrast pleas-decide - rate ( $\left.\left[\begin{array}{lll}48 & 26 & 4\end{array}\right], z=3.69, p=.002\right)$, with the time course shown in panel C. (B) A relatively anterior part of the insula was activated in the contrast intens-decide - rate ([ $\left.\begin{array}{lll}42 & 12 & 0\end{array}\right]$, $z=3.56, p=.004)$, with the time course shown in panel $\mathrm{D}$, implicating this part of the insula particularly in decisions about the physical properties of stimuli, such as intensity. (E) There was a significant interaction between the SPM parameter estimates (arbitrary units) for the activations in the agranular insula versus the anterior insula when taking decisions about pleasantness versus intensity, $F(1,11)=15.3$, $p=.002$.

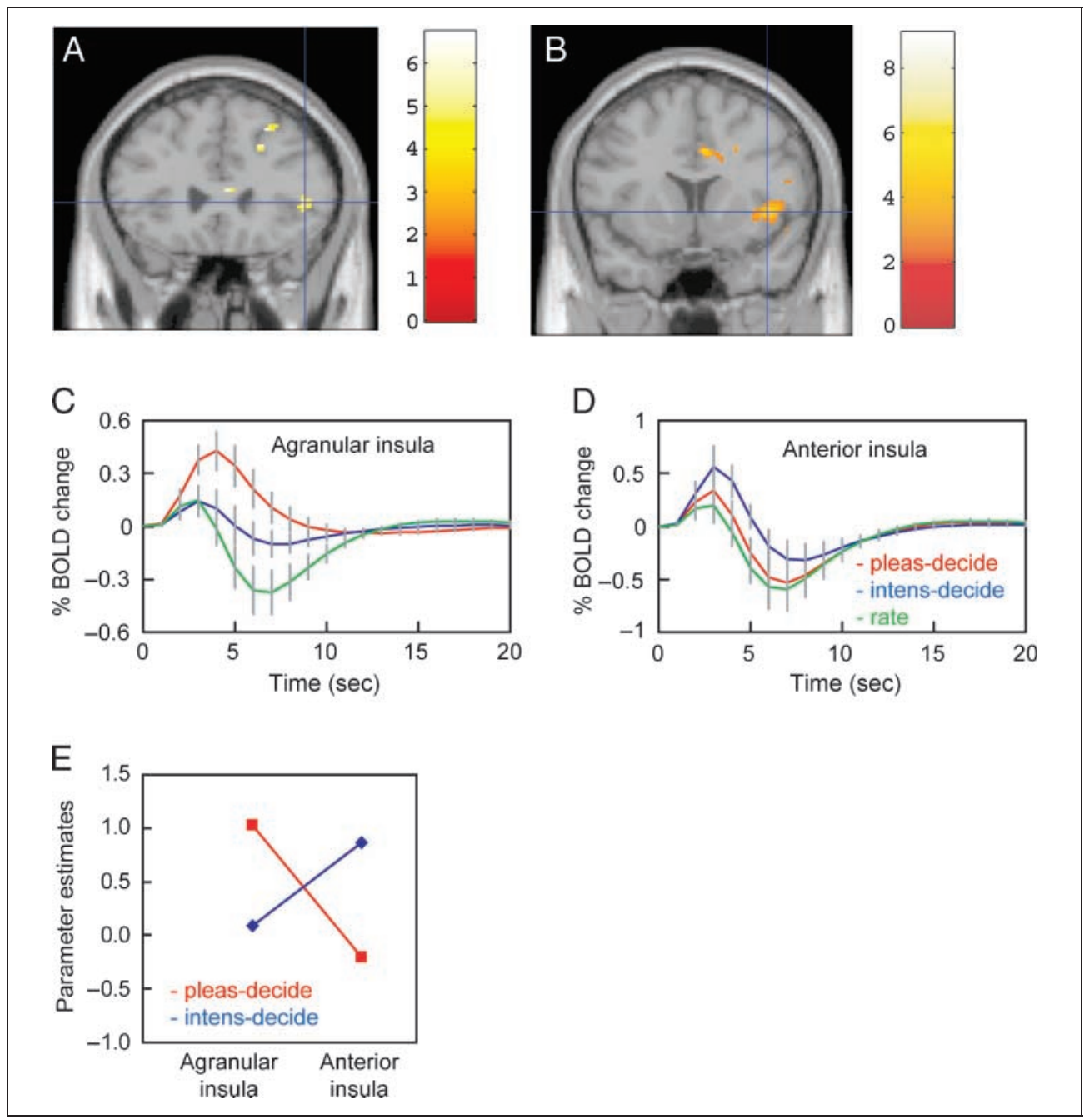


related to affective processing (Grabenhorst, Rolls, \& Parris, 2008; Grabenhorst et al., 2007).

\section{Dorsal Anterior Cingulate Cortex}

A contrast of all trials on which decisions were made versus all trials on which ratings were made (the contrast alldecide - rate) revealed statistically significant effects in the SPM analysis in dorsal ACC (Figure 2A, white circle). This region did not show correlations with the intensity or the pleasantness ratings, hence may be related more to the decision-making than to the representation of affective value. Further, the BOLD signals in this brain region were related to both types of decision, in that there were significant effects in the SPM contrast pleas-decide rate ([6 2612$], z=3.81, p=.007$ ) and in the contrast intens-decide - rate ([1 $\left.\left.\begin{array}{lll}10 & 30 & 28\end{array}\right], z=3.45, p=.003\right)$. These findings are illustrated by the time courses of the BOLD signal (mean across subjects \pm SEM) shown in Figure 2C for the different conditions. It is shown that with a typical hemodynamic response that would peak 5-7 sec after the second odor, the BOLD signal on both types of decision trial is higher than on the rating trials. In olfactory tasks with a delay between stimuli, a reduction in the BOLD signal can occur after an odor has been delivered (Gottfried, Winston, \& Dolan, 2006), but our finding was that the BOLD signal was relatively much higher if it was a decision compared with a rating trial, as illustrated in Figure 2C.

Dorsal ACC showed more activation on difficult than on easy trials, consistent with the possibility that difficult decisions require more attention or conflict, which engage this brain region (Greene, Nystrom, Engell, Darley, \& Cohen, 2004). In particular, an SPM analysis performed on the decision trials in which the regressor was the absolute value of each subject's average difference in the pleasantness of each pair of stimuli (as obtained from the rating trials) showed a significant negative correlation in dorsal ACC ( 030 10], $z=2.72, p=.045)$.

\section{Ventral Premotor Cortex}

Decisions about intensity versus decisions about pleasantness (intens-decide - pleas-decide) revealed significant effects in the ventral premotor cortex (Figure 6, at cursor). There was also a significant effect in the contrast intens-decide - rate at [56 2 36], $z=4.50, p<.001$.

\section{Ventral Tegmental Area}

Significant effects were found for the contrast pleas-deciderate in ventral tegmental area, in a region close to the dopamine neurons ([2-10-8], $z=2.96, p=.023)$, implicating this region in decisions about pleasantness. Consistent with this, there was an effect in the contrast pleas-decide - intens-decide, $[10-12-4]$, as shown in Table 1 in the Supplementary material. This pattern of results in decision-making is consistent with effects found when decisions must be made about pleasant warm stimuli versus unpleasant cold stimuli applied to the hand (Grabenhorst, Rolls, \& Parris, 2008).

The effects in the above regions on the decision trials were not related to movements in the response period of the task, based on the following evidence. First, the effects were found at $t=8 \mathrm{sec}$ in the trial when the second odor was delivered, and the cue to allow a button keypress to select the response was not shown until $t=16 \mathrm{sec}$. Second, the contrasts shown in Figure 2 were exclusively masked by the activations occurring when the response was made at $t=16 \mathrm{sec}$. These movement-related activations are shown in Figure 7 and were in different brain regions such as the primary motor cortex area 4 . Further, the same type of response, using the button box, was required on decision and rating trials, so responses per se did not contribute to the different signal changes found (earlier in the trial) on decide versus rate trials.

\section{DISCUSSION}

In this study, significantly larger BOLD signals were found in medial prefrontal cortex area 10 when a decision had to be made compared with when the same affective olfactory stimuli had to be rated for affective value and intensity. This was shown in a contrast analysis (Figure 2A, pink circle). The implication is that this part of medial area 10 contributes in some way beyond representing affective value to the decision-making process. Further, the signals in medial area 10 were not correlated with the pleasantness (or intensity) ratings, again supporting the hypothesis that
Figure 6. Decisions about intensity compared with decisions about pleasantness The contrast intens-decide pleas-decide showed significant effects in ventral premotor cortex, [58 $\left.4 \begin{array}{ll}52 & 22\end{array}\right], z=3.30$, $p=.014$.
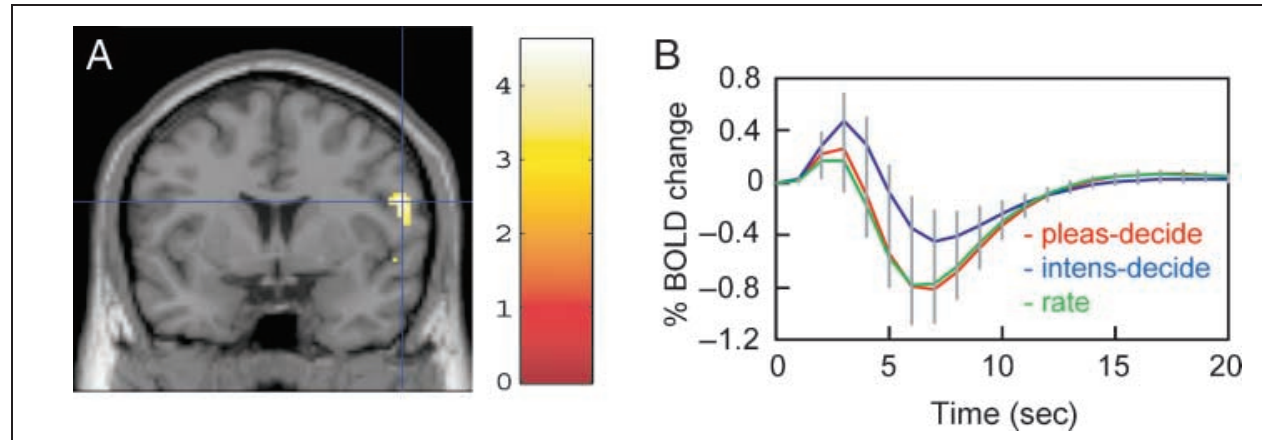


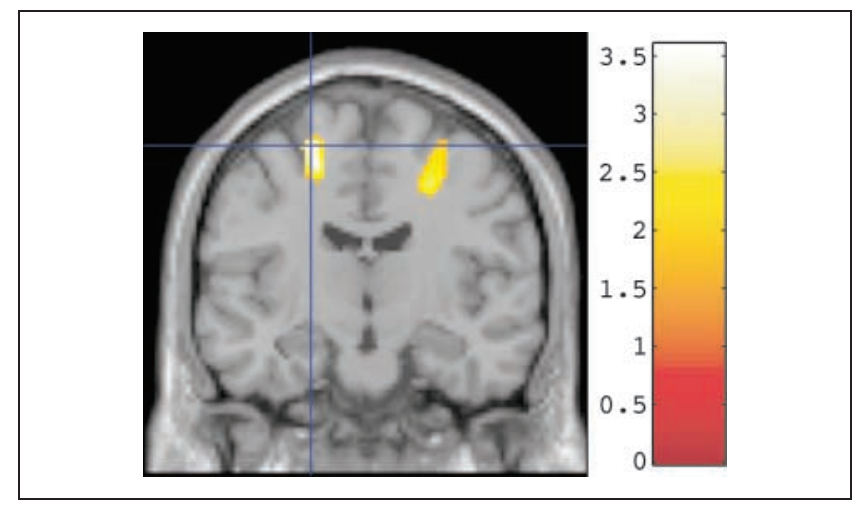

Figure 7. Response-related activation: Activations related to the responses made with the button box at $t=16 \mathrm{sec}$ when responses were made to indicate a rating. Significant effects were found in the motor cortex area $4,[-22-1262], z=2.88, p=.034$.

activity in medial area 10 is more closely related to decisionmaking than to the representation of affective value. The effects found in medial area 10 were anterior to pregenual cingulate cortex (Figure 2) where activations for at least some stimuli are related to their pleasantness (see Introduction). At least part, the more dorsal part, of medial area 10 had signals that were especially related to decisions about pleasantness, as shown by the contrast pleas-decide intens-decide (Figure 4B), and even this part of medial area 10 did not have signals that were correlated with pleasantness. Part of the interest of this finding is that the stimuli being delivered on the rating and decision trials are identical, so that what has been revealed by the effects in medial prefrontal cortex area 10 is of processing especially related to decision-making compared with only rating the stimuli.

We found that the medial area 10 BOLD signal related to decision-making versus rating extended down into a part of medial OFC, as shown in Figure 2A (yellow circle). The activations here were not related to the pleasantness of the olfactory stimuli. The interesting implication of the new finding described here is that at least parts of medial OFC may be involved in decision-making and not in representing the affective value of the stimuli or may receive feedback connections from brain regions such as medial area 10 (Petrides \& Pandya, 2007) implicated in the decision-making.

What other evidence is there that medial prefrontal cortex area 10 may make a special contribution to decisionmaking? It has been shown in patients with medial prefrontal cortex lesions that decision-making in a shopping task is impaired, with subjects having difficulty in the decision-making as reflected for example by visits to previously visited locations (Burgess et al., 2007; Burgess, 2000; Shallice \& Burgess, 1991). Decision-making impairments are also found in patients with ventromedial prefrontal cortex lesions (Bechara et al., 2000), although these lesions are large, and OFC may be a more crucial site for impairments in altering choices when reinforcement contingencies change (Rolls, 2008; Hornak et al., 2004; Fellows \& Farah,
2003; Rolls, Hornak, Wade, \& McGrath, 1994). It is of interest that the effects described here related to decisionmaking were found when the decision-making was about simple affective stimuli, olfactory stimuli (Rolls, 2008).

In comparison, the mid OFC area shown in Figure 3 is not implicated in the processing that is special to taking the decision (as shown by no effect in the contrast alldecide - rate; see parameter estimates close to zero in Figure 3D), but it is implicated in the continuous affective representation of the stimuli (as shown by the correlation with the pleasantness ratings; Figure 3B). Indeed, the data in Figure 3B show that for this region, the percentage of change in the BOLD signal varies continuously with the value of the pleasantness rating. The parameter estimates shown in Figure 3D and the significant interaction effect in the ANOVA show that this mid OFC region has activations related to pleasantness ratings and not to the binary decisions about the odor, whereas the medial prefrontal cortex region has signals related to the binary decision-making but not to the continuous affective value of the stimuli and that the dissociation is significant $(p<$ .002). A function of mid (and frequently medial) OFC in representing affective value is consistent with studies showing that it has activations that are continuously related to the affective value of monetary, somatosensory, olfactory, and taste stimuli (see Introduction), and the new finding here is that it does not show further activation when a decision is being made.

An anterior part of insula ( $y=12$; Figure 5B) had activations related to decisions about intensity versus only rating, whereas agranular (far anterior) insula ( $y=26$; Figure $5 \mathrm{~A}$ ) had activations related to decisions about pleasantness versus only rating (Figure 5), suggesting that affective decision-making recruits the far anterior (agranular) insula, which is activated by taste-olfactory convergence that can contribute to making a flavor pleasant (de Araujo, Rolls, Kringelbach, McGlone, \& Phillips, 2003) and which might be involved in autonomic effects resulting from the decision (Critchley, Wiens, Rotshtein, Ohman, \& Dolan, 2004). Interestingly, there are projections from area 10 to insula (Petrides \& Pandya, 2007), and these are a possible source of the decision-related activations described in the current investigation.

The dorsal part of the ACC was also implicated more in decision-making than in representing affective value in this investigation (Figure 2A, white circle) and indeed in decision-making about both pleasantness and intensity. With respect to ACC, we note that this has been implicated in action-outcome learning (Rushworth, Behrens, et al., 2007; Rushworth, Buckley, Behrens, Walton, \& Bannerman, 2007), and its activation described here is consistent with a role of the cingulate cortex in decisionmaking. In a further analysis, the dorsal ACC region showed more activation on difficult than on easy trials, consistent with the possibility that difficult decisions require more attention or conflict, which engage this brain region (Greene et al., 2004). 
Part of the strength of the design in the present article is the within-subjects design, and this leads to the result that decision-making parts of the medial prefrontal cortex are anterior to the region further posterior in the mid OFC where there is a correlation with the pleasantness ratings.

In medial prefrontal areas in which the BOLD signal was larger during choice decision-making than during ratings of continuous value, it was the case that even during decision-making, some deactivation was found. It has been noted by others (Summerfield \& Koechlin, 2008) that in delay matching tasks, signals in ventromedial prefrontal cortex, OFC, and cingulate cortex are lower during the task than in baseline conditions between trials, although interesting differences between match and nonmatch conditions are found. Also it is a property that the baseline activation level in some brain regions can be high in the resting state, and against this background, deactivations can be found (Morcom \& Fletcher, 2007), which may be task dependent, as in the present study. Although medial prefrontal areas were somewhat deactivated during decision-making (see Figure 2), the signal in this brain region was much lower when choice decisions were not being made, and continuous value was being assessed. Moreover, the signal present in medial prefrontal cortex during decision-making does appear to be relevant to decision-making because lesions of medial prefrontal cortex in humans impair decision-making (Burgess et al., 2007; Burgess, 2000; Shallice \& Burgess, 1991). In addition, the effects found are of interest because they suggest that it would be of interest to examine medial prefrontal cortex area 10 in decision tasks such as those used by de Lafuente and Romo (2006) and Romo et al. (2004), in which the overall design with two stimuli separated by a delay after which a decision must be made is analogous. Indeed, given the significant differences found in medial area 10 in this study related to whether a choice decision is made or not, it would be very interesting to examine exactly how neuronal activity in this region is related to decision-making.

The implication of the findings described here is that whereas areas such as parts of OFC (Rolls, Grabenhorst, $\&$ Parris, 2008) provide a continuous representation of the value of affective stimuli, medial prefrontal cortex area 10 is involved beyond the evaluation of affective stimuli in the decision-making process itself. For the evaluation, the neural activity needs to represent a stimulus in a way that continuously and faithfully represents the affective value of the stimulus, and this could be present independently of whether a binary decision is being made or not. On the other hand, when a binary decision must be reached, the neural activity needs no longer to continuously represent the affective value of the stimulus but needs to fall into a state in which the high firing of some neurons represents one decision or the high firing of other neurons a different decision. Processes such as this transition to a binary state of firing of neurons (fast vs. slow) are known to occur in some premotor and related areas such as macaque ventral premotor cortex (de Lafuente \& Romo, 2006; Romo et al., 2004). In the present study, we found that activation of the human ventral premotor cortex was greater for decisions about the intensity than for decisions about the pleasantness of olfactory stimuli. The evidence described here thus indicates that human medial prefrontal cortex area 10 may be particularly involved in this special transition that is thought to occur as the binary decision is being reached (Rolls, 2008; Deco \& Rolls, 2006; Wang, 2002). There is further evidence for this. Noting that on easy trials of a decision task neurons respond with shorter latencies than on difficult trials (Shadlen \& Newsome, 2001; Kim \& Shadlen, 1999), Heekeren, Marrett, and Ungerleider (2008) and Heekeren et al. (2004) have used a comparison of easy versus difficult trials in human fMRI studies to identify areas involved in decision-making. In addition, the attractor network model of decision-making falls into one of its high firing rate attractor states earlier on easy than difficult trials (Deco \& Rolls, 2006), and this is predicted to produce a larger BOLD signal on easy than on difficult trials. As will be reported elsewhere with corresponding model simulation results, using a contrast of easy versus difficult trials, with the current data set, we found significant effects for the medial prefrontal cortex area 10 ([4 52 $-14], z=3.44, p=.007$ ), which corresponds closely and confirms the results reported in this article. (For this analysis, the easy trials were those where the first and second odors were by design very different in pleasantness, e.g., citral and isovaleric acid, and the difficult trials were those where by design the first and second odors were both from the pleasant category, or both from the unpleasant category.) The "easy" trials were indeed easy in that the mean choice value of one versus the other odor was $90 \pm 3 \%$ (mean $\pm S E M)$, whereas for the "difficult" trials, the mean choice values were $60 \pm 8 \%$ (paired $t$ test, $p=.014$ ). A finite impulse response model was used as we had a prediction that the effect should be clear soon after the second odor was presented, and the statistics just provided were for a time that was 2-4 sec after the onset of the second odor, which is when the decision was being taken. This is useful confirmation of the results described here. In addition, as shown in Figure 2E, the activations in this medial prefrontal cortex area 10 region were correlated with the easiness of the decisions about intensity. No significant effects of this type of analysis of task easiness were found in the OFC regions described here, where there was a correlation of the BOLD signal with the continuous-valued pleasantness ratings.

Areas such as DLPFC have been implicated by earlier studies in decision-making (Preuschhof, Heekeren, Taskin, Schubert, \& Villringer, 2006; Heekeren et al., 2004; Kim \& Shadlen, 1999). However, an interesting finding made here is that whereas these areas may be especially related to decision-making about the physical properties of stimuli such as their intensity, other brain regions such as the more dorsal parts of medial prefrontal cortex area 10 (see Figure 2) may be more closely involved in affective 
decision-making. Further, activations in DLPFC, dorsal ACC, and agranular insula were related to the difficulty of the decisions as shown by negative correlations with the absolute difference in subjective intensity or pleasantness of each pair of stimuli, so that their engagement in decision-making tasks may be related to greater attentional or emotional processing on difficult trials. In contrast, the greater activation of the medial prefrontal cortex area 10 on easy versus difficult trials described here implicates it much more directly in the process by which a network falls into an attractor state representing a decision more rapidly on easy trials (Deco et al., 2009; Deco \& Rolls, 2006).

\section{Acknowledgments}

F. G. was supported by the Gottlieb-Daimler and Karl BenzFoundation, and by the Oxford Centre for Computational Neuroscience. The investigation was performed at the Centre for Functional Magnetic Resonance Imaging of the Brain (FMRIB) at Oxford University.

Reprint requests should be sent to Professor Edmund T. Rolls, Oxford Centre for Computational Neuroscience, Oxford, UK, or via e-mail: Edmund.Rolls@oxcns.org, Web site: http://www. oxcns.org.

\section{REFERENCES}

Anderson, A. K., Christoff, K., Stappen, I., Panitz, D., Ghahremani, D. G., Glover, G., et al. (2003). Dissociated neural representations of intensity and valence in human olfaction. Nature Neuroscience, 6, 196-202.

Bechara, A., Damasio, H., \& Damasio, A. R. (2000). Emotion, decision making and the orbitofrontal cortex. Cerebral Cortex, 10, 295-307.

Behrens, T. E., Woolrich, M. W., Walton, M. E., \& Rushworth, M. F. (2007). Learning the value of information in an uncertain world. Nature Neuroscience, 10, 1214-1221.

Burgess, P. W. (2000). Strategy application disorder: The role of the frontal lobes in human multitasking. Psychological Research, 63, 279-288.

Burgess, P. W., Dumontheil, I., \& Gilbert, S. J. (2007). The gateway hypothesis of rostral prefrontal cortex (area 10) function. Trends in Cognitive Sciences, 11, 290-298.

Collins, D. L., Neelin, P., Peters, T. M., \& Evans, A. C. (1994). Automatic 3D intersubject registration of MR volumetric data in standardized Talairach space. Journal of Computer Assisted Tomography, 18, 192-205.

Critchley, H. D., Wiens, S., Rotshtein, P., Ohman, A., \& Dolan, R. J. (2004). Neural systems supporting interoceptive awareness. Nature Neuroscience, 7, 189-195.

de Araujo, I. E. T., Kringelbach, M. L., Rolls, E. T., \& Hobden, P. (2003). The representation of umami taste in the human brain. Journal of Neurophysiology, 90, 313-319.

de Araujo, I. E. T., Rolls, E. T., Kringelbach, M. L., McGlone, F., \& Phillips, N. (2003). Taste-olfactory convergence, and the representation of the pleasantness of flavour, in the human brain. European Journal of Neuroscience, 18, 2374-2390.

de Araujo, I. E. T., Rolls, E. T., Velazco, M. I., Margot, C., \& Cayeux, I. (2005). Cognitive modulation of olfactory processing. Neuron, 46, 671-679. de Lafuente, V., \& Romo, R. (2006). Neural correlate of subjective sensory experience gradually builds up across cortical areas. Proceedings of the National Academy of Sciences, U.S.A., 103, 14266-14271.

Deco, G., \& Rolls, E. T. (2006). Decision-making and Weber's Law: A neurophysiological model. European Journal of Neuroscience, 24, 901-916.

Deco, G., Rolls, E. T., \& Romo, R. (2009). Stochastic dynamics as a principle of brain function. Progress in Neurobiology, doi:10.1016/j.pneurobio.2009.01.006.

Fellows, L. K., \& Farah, M. J. (2003). Ventromedial frontal cortex mediates affective shifting in humans: Evidence from a reversal learning paradigm. Brain, 126, 1830-1837.

Friston, K. J., Glaser, D. E., Henson, R. N., Kiebel, S., Phillips, C., \& Ashburner, J. (2002). Classical and Bayesian inference in neuroimaging: Applications. Neuroimage, 16, 484-512.

Friston, K. J., Worsley, K. J., Frackowiak, R. S. J., Mazziotta, J. C., \& Evans, A. C. (1994). Assessing the significance of focal activations using their spatial extent. Human Brain Mapping, 1, 214-220.

Glimcher, P. W. (2003). The neurobiology of visual-saccadic decision making. Annual Review of Neuroscience, 26, 133-179.

Gottfried, J. A., Winston, J. S., \& Dolan, R. J. (2006). Dissociable codes of odor quality and odorant structure in human piriform cortex. Neuron, 49, 467-479.

Grabenhorst, F., Rolls, E. T., \& Bilderbeck, A. (2008). How cognition modulates affective responses to taste and flavor: Top down influences on the orbitofrontal and pregenual cingulate cortices. Cerebral Cortex, 18, 1549-1559.

Grabenhorst, F., Rolls, E. T., Margot, C., da Silva, M. A. A. P., \& Velazco, M. I. (2007). How pleasant and unpleasant stimuli combine in different brain regions: Odor mixtures. Journal of Neuroscience, 27, 13532-13540.

Grabenhorst, F., Rolls, E. T., \& Parris, B. A. (2008). From affective value to decision-making in the prefrontal cortex. European Journal of Neuroscience, 28, 1930-1939.

Greene, J. D., Nystrom, L. E., Engell, A. D., Darley, J. M., \& Cohen, J. D. (2004). The neural bases of cognitive conflict and control in moral judgment. Neuron, 44, 389-400.

Guest, S., Grabenhorst, F., Essick, G., Chen, Y., Young, M., McGlone, F., et al. (2007). Human cortical representation of oral temperature. Physiology and Behavior, 92, 975-984.

Hampton, A. N., \& O'Doherty, J. P. (2007). Decoding the neural substrates of reward-related decision making with functional MRI. Proceedings of the National Academy of Sciences, U.S.A., 104, 1377-1382.

Heekeren, H. R., Marrett, S., Bandettini, P. A., \& Ungerleider, L. G. (2004). A general mechanism for perceptual decisionmaking in the human brain. Nature, 431, 859-862.

Heekeren, H. R., Marrett, S., \& Ungerleider, L. G. (2008). The neural systems that mediate human perceptual decision making. Nature Reviews. Neuroscience, 9, 467-479.

Heekeren, H. R., Wartenburger, I., Schmidt, H., Prehn, K., Schwintowski, H. P., \& Villringer, A. (2005). Influence of bodily harm on neural correlates of semantic and moral decision-making. Neuroimage, 24, 887-897.

Hornak, J., Bramham, J., Rolls, E. T., Morris, R. G., O’Doherty, J., Bullock, P. R., et al. (2003). Changes in emotion after circumscribed surgical lesions of the orbitofrontal and cingulate cortices. Brain, 126, 1691-1712.

Hornak, J., O’Doherty, J., Bramham, J., Rolls, E. T., Morris, R. G., Bullock, P. R., et al. (2004). Reward-related reversal learning after surgical excisions in orbitofrontal and dorsolateral prefrontal cortex in humans. Journal of Cognitive Neuroscience, 16, 463-478.

Kiebel, S. J., Poline, J. B., Friston, K. J., Holmes, A. P., \& Worsley, K. J. (1999). Robust smoothness estimation in statistical 
parametric maps using standard residuals from the general linear model. Neuroimage, 10, 756-766.

Kim, J. N., \& Shadlen, M. N. (1999). Neural correlates of a decision in the dorsolateral prefrontal cortex of the macaque. Nature Neuroscience, 2, 176-185.

Knutson, B., Rick, S., Wimmer, G. E., Prelec, D., \& Loewenstein, G. (2007). Neural predictors of purchases. Neuron, 53, 147-156.

Kringelbach, M. L., \& Rolls, E. T. (2003). Neural correlates of rapid reversal learning in a simple model of human social interaction. Neuroimage, 20, 1371-1383.

Lau, H. C., \& Passingham, R. E. (2007). Unconscious activation of the cognitive control system in the human prefrontal cortex. Journal of Neuroscience, 27, 5805-5811.

Marsh, A. A., Blair, K. S., Vythilingam, M., Busis, S., \& Blair, R. J. (2007). Response options and expectations of reward in decision-making: The differential roles of dorsal and rostral anterior cingulate cortex. Neuroimage, 35, 979-988.

Moll, J., Krueger, F., Zahn, R., Pardini, M., de Oliveira-Souza, R., \& Grafman, J. (2006). Human fronto-mesolimbic networks guide decisions about charitable donation. Proceedings of the National Academy of Sciences, U.S.A., 103, 15623-15628.

Morcom, A. M., \& Fletcher, P. C. (2007). Does the brain have a baseline? Why we should be resisting a rest. Neuroimage, 37, 1073-1082.

Morris, G., Nevet, A., Arkadir, D., Vaadia, E., \& Bergman, H. (2006). Midbrain dopamine neurons encode decisions for future action. Nature Neuroscience, 9, 1057-1063.

Ochsner, K. N., Knierim, K., Ludlow, D. H., Hanelin, J., Ramachandran, T., Glover, G., et al. (2004). Reflecting upon feelings: An fMRI study of neural systems supporting the attribution of emotion to self and other. Journal of Cognitive Neuroscience, 16, 1746-1772.

O’Doherty, J., Kringelbach, M. L., Rolls, E. T., Hornak, J., \& Andrews, C. (2001). Abstract reward and punishment representations in the human orbitofrontal cortex. Nature Neuroscience, 4, 95-102.

O’Doherty, J., Rolls, E. T., Francis, S., Bowtell, R., \& McGlone, F. (2001). The representation of pleasant and aversive taste in the human brain. Journal of Neurophysiology, 85, 1315-1321.

O'Doherty, J., Winston, J., Critchley, H., Perrett, D., Burt, D. M., \& Dolan, R. J. (2003). Beauty in a smile: The role of medial orbitofrontal cortex in facial attractiveness. Neuropsychologia, 41, 147-155.

Petrides, M., \& Pandya, D. N. (2007). Efferent association pathways from the rostral prefrontal cortex in the macaque monkey. Journal of Neuroscience, 27, 11573-11586.

Preuschhof, C., Heekeren, H. R., Taskin, B., Schubert, T., \& Villringer, A. (2006). Neural correlates of vibrotactile working memory in the human brain. Journal of Neuroscience, 26, 13231-13239.

Rolls, E. T. (2008). Memory, attention, and decision-making: A unifying computational neuroscience approach. Oxford: Oxford University Press.

Rolls, E. T., Critchley, H. D., Mason, R., \& Wakeman, E. A. (1996). Orbitofrontal cortex neurons: Role in olfactory and visual association learning. Journal of Neurophysiology, 75, 1970-1981.

Rolls, E. T., \& Grabenhorst, F. (2008). The orbitofrontal cortex and beyond: From affect to decision-making. Progress in Neurobiology, 86, 216-244.

Rolls, E. T., Grabenhorst, F., \& Parris, B. A. (2008). Warm pleasant feelings in the brain. Neuroimage, 41, 1504-1513.
Rolls, E. T., Hornak, J., Wade, D., \& McGrath, J. (1994). Emotion-related learning in patients with social and emotional changes associated with frontal lobe damage. Journal of Neurology, Neurosurgery and Psychiatry, 57, 1518-1524

Rolls, E. T., Kringelbach, M. L., \& de Araujo, I. E. T. (2003). Different representations of pleasant and unpleasant odors in the human brain. European Journal of Neuroscience, 18, 695-703.

Rolls, E. T., O’Doherty, J., Kringelbach, M. L., Francis, S., Bowtell, R., \& McGlone, F. (2003). Representations of pleasant and painful touch in the human orbitofrontal and cingulate cortices. Cerebral Cortex, 13, 308-317.

Rolls, E. T., Sienkiewicz, Z. J., \& Yaxley, S. (1989). Hunger modulates the responses to gustatory stimuli of single neurons in the caudolateral orbitofrontal cortex of the macaque monkey. European Journal of Neuroscience, 1, 53-60.

Romo, R., Hernandez, A., \& Zainos, A. (2004). Neuronal correlates of a perceptual decision in ventral premotor cortex. Neuron, 41, 165-173.

Rushworth, M. F., Behrens, T. E., Rudebeck, P. H., \& Walton, M. E. (2007). Contrasting roles for cingulate and orbitofrontal cortex in decisions and social behaviour. Trends in Cognitive Sciences, 11, 168-176.

Rushworth, M. F., Buckley, M. J., Behrens, T. E., Walton, M. E., \& Bannerman, D. M. (2007). Functional organization of the medial frontal cortex. Current Opinion in Neurobiology, 17, 220-227.

Shadlen, M. N., \& Newsome, W. T. (2001). Neural basis of a perceptual decision in the parietal cortex (area LIP) of the rhesus monkey. Journal of Neurophysiology, 86, 1916-1936.

Shallice, T., \& Burgess, P. W. (1991). Deficits in strategy application following frontal lobe damage in man. Brain, 114, 727-741.

Small, D. M., Gregory, M. D., Mak, Y. E., Gitelman, D., Mesulam, M. M., \& Parrish, T. (2003). Dissociation of neural representation of intensity and affective valuation in human gustation. Neuron, 39, 701-711.

Spitzer, M., Fischbacher, U., Herrnberger, B., Gron, G., \& Fehr, E. (2007). The neural signature of social norm compliance. Neuron, 56, 185-196.

Sugrue, L. P., Corrado, G. S., \& Newsome, W. T. (2005). Choosing the greater of two goods: Neural currencies for valuation and decision making. Nature Reviews. Neuroscience, 6, 363-375.

Summerfield, C., \& Koechlin, E. (2008). A neural representation of prior information during perceptual inference. Neuron, 59, 336-347.

Thielscher, A., \& Pessoa, L. (2007). Neural correlates of perceptual choice and decision making during fear-disgust discrimination. Journal of Neuroscience, 27, 2908-2917.

Wang, X. J. (2002). Probabilistic decision making by slow reverberation in cortical circuits. Neuron, 36, 955-968.

Wilson, J. L., Jenkinson, M., Araujo, I. E. T., Kringelbach, M. L., Rolls, E. T., \& Jezzard, P. (2002). Fast, fully automated global and local magnetic field optimisation for fMRI of the human brain. Neuroimage, 17, 967-976.

Worsley, K. J., Marrett, P., Neelin, A. C., Friston, K. J., \& Evans, A. C. (1996). A unified statistical approach for determining significant signals in images of cerebral activation. Human Brain Mapping, 4, 58-73.

Zatorre, R. J., Jones-Gotman, M., \& Rouby, C. (2000). Neural mechanisms involved in odour pleasantness and intensity judgements. NeuroReport, 11, 2711-2716. 